\title{
Fuzzy Logic Based Intelligent Temperature Controller for Cassava Post-Harvest Storage System
}

\author{
Adamu Y. Babawuro ${ }^{1,2}$, Sambo A. Umar ${ }^{1,2}$, Sado Fatai ${ }^{1}$, Momoh Jimoh E. Salami ${ }^{1}$ \\ and Shahrul Naim Sidek ${ }^{1}$ \\ ${ }^{1}$ Mechatronics Engineering Department, Kulliyyah of Engineering, \\ International Islamic University Malaysia (IIUM), Kuala Lumpur, Malaysia. \\ ${ }^{2}$ Mechatronics and Systems Engineering Department, Faculty of Engineering, \\ Abubakar Tafawa Balewa University (ATBU), Bauchi, Bauchi State, Nigeria.
}

\begin{abstract}
Significant amount of stored agricultural products are lost as a result of poor and inefficient storage systems in most developing countries, especially in tropical regions of the world. Improvements on the existing storage methods is important to guarantee food security. This study proposes the development of intelligent temperature control technique for fresh cassava roots crop post-harvest storage system using fuzzy logic controller (FLC). The intelligent controller which has two inputs (error in temperature and rate of change in the error) and one output (change in fan speed) was simulated with the developed storage system model for temperature control of fresh cassava roots crop. The results obtained shows that the controller can track appropriately the reference temperature and also gives good stability and robustness towards input disturbances. Faster response to maintain the storage temperature within acceptable limit close to reference point was also achieved successfully.
\end{abstract}

Keywords: Post-Harvest, Storage, Cassava, Temperature, Intelligent Control, FLC

\section{Introduction}

Post-harvest storage systems for agricultural products are usually employed to maintain quality of agricultural products by decelerating the ripening process, reducing respiratory heat emission, and curbing moisture loss due to transpiration and spoilage as a result of microbial activities [1]. Recent statistics shows that, between $25-40 \%$ of stored agricultural products goes into waste each year due to insufficient storage facilities. The products may be exposed to insects, rodents, birds and other pests. They may also be spoiled by fungi, yeast and bacteria. Therefore, knowing the optimum environmental conditions for storage product and the conditions under which the micro-organisms grow is of enormous importance in order to minimize losses during storage [2]. Improvements on existing post-harvest methods is important in ensuring food security. In recent years, the importance has been pointed-out as can be evident in the Food and Agricultural Organization (FAO) Cooperate Document Repository (2010) [3].

During storage, more especially in confined environment, most tropical root crops such as yam tuber and cassava root releases moisture, heat, ethylene and carbon dioxide gases as a result of the physiological processes occurring within the body of the produce. These physiological activities are sprouting, transpiration and respiration which depend largely on temperature and relative humidity of storage environment. These emissions from the body of the agricultural product can be harmful if not handled properly[2, 4]. This results in limited acidity level of the products and thereby affecting its edible content, nutritional and cooking qualities and also market prices[5]. In rural areas, this was not a problem as the farmers harvest and process the roots almost immediately for either consumption or commercialization and, hence, the risk of post-harvest deterioration is reduced significantly. Post-harvest storage of agricultural products is an important part of food processing that guarantees food security by ensuring its availability even in scarcity period [3,5]. Hence, there is a demand for optimum control of storage temperature and relative humidity. 
Cassava (Manihot esculenta Crantz) is the most important tropical root crop in West Africa[4]. The production of cassava is perennial and grows primarily in tropical and subtropical regions of the world. It is a major staple crop in tropical regions that can be consumed in raw form, baked, boiled or usually fermented into other foods and beverages all over the globe. In developing countries, cold storage of fresh cassava roots is usually not feasible as a result of financial status and technical knowledge of most local farmers. Typical cassava roots crop is illustrated in Fig. 1.

Temperature and relative humidity of storage environment as well as moisture content of cassava roots determines better storage of the product. According to [5], cassava roots stored best at a temperature of $3^{\circ} \mathrm{C}$, with a total weight loss of $14 \%$ after two weeks and $23 \%$ after four weeks of storage. At storage temperature below $3^{\circ} \mathrm{C}$, cassava roots shows internal browning disorder, while it manifests faster rate of physiological deterioration when the storage temperature is raised to room temperature. Maintaining optimum storage conditions (temperature and relative humidity) for stored cassava product is therefore considered paramount to achieving good storage objectives.

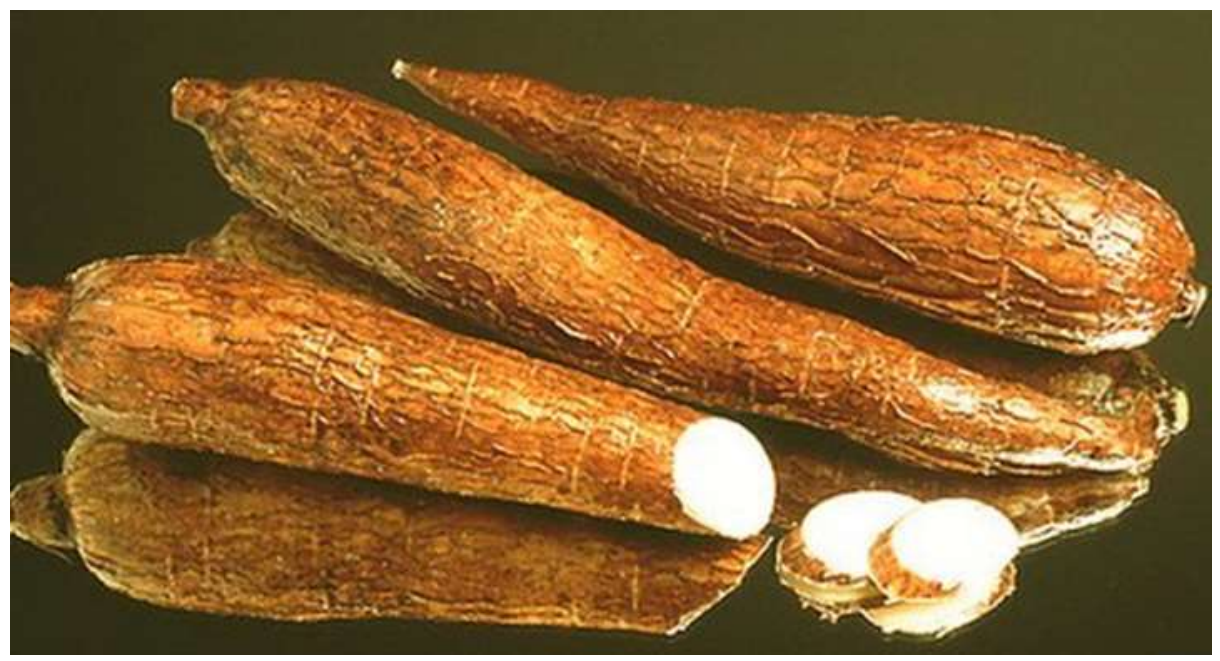

Fig. 1: Typical Cassava Roots.

It has been shown that, several indigenous storage techniques have been employed for preservation and reduction of cassava post-harvest losses. Although, they have been successful in prolonging the shelf life of the root for some few days [5], studies shows that they have serious limitations. The easiest and simplest traditional cassava root crop storage method is in-ground storage. In this method, matured roots are left un-harvested beneath the earth until immediate need such as commercialization, consumption or processing arises. Although, the root can last up to three years, the major drawback of this storage technique is that large area of land is used by the buried roots, making it unavailable for further agricultural production. In addition, the root may increase in size and become more fibrous and woody, decreasing in palatability and increasing in cooking period if not harvested in optimal time. They may also be more prone to attacks by pathogens and rodents as well as limited extractable starch [6]. Another method is burying the roots in the soil and stacking the roots in piles and ensuring constant watering. This method is not safe as root deterioration could begin from the bottom root. Several attempts have been made in order to improve on the indigenous storage techniques for cassava root crops with the aim of restraining deterioration and extending their shelf life. These includes the use of techniques such as Field clamp storage, pit storage, storing the cassava roots crop in boxes lined with wood shavings or moist sawdust and storage in plastic film wraps or plastic bags [5]. Studies have shown, however, that these techniques can only extend the shelf life of the root to about four to eight weeks with a considerable amount of losses ranging from $25 \%$ to $50 \%$ of the products.

Furthermore, some advanced techniques have been proposed by National Resources Institute (NRI), Chatham, UK, in collaboration with Centro Internacional de Agricultura Tropical (CIAT) Palmira, Colombia. 
The proposed methods are refrigeration at lower temperature within the limit of $0-5^{\circ} \mathrm{C}$, waxing with paraffin wax and freeze drying which are primarily employed for exportation of the cassava roots. These techniques have been reported to have improved the storage of fresh cassava root by about two weeks without any loss reported. However, refrigeration is not commonly employed in developing countries and maintenance of the equipment for this purpose are costly, and needs skilled expertise to handle. Refrigeration cooling method is mostly used in post-harvest storage system. This include: forced-air cooling, natural convective cooling, vacuum cooling, mistflow cooling, adiabatic cooling, hydro cooling and ice cooling. However, the most commonly employed cooling method is forced-air cooling technique. This is because of its low operational cost, better efficiency and flexibility [1, 6]. Forced-air cooling systems mostly comprises of a cooling unit assembly coupled with cooling coils in which the refrigerant is circulated and a suction fan that draws (forces) cooling air over the coils through the products [7]. In this study, a simple, efficient, affordable and cost effective forced-air fresh cassava roots storage system using fuzzy logic control approach is proposed. The FLC takes-in two inputs (error and error change in temperature) and uses this to regulate the speed of the two fans (cooling and circulating) appropriately based on sensed fluctuations in air temperature within the storage compartments. The regulated FLC output is directed as control input to the cooling unit which is then produce the desired air temperature for the storage compartments.

Section 2 of this paper presents the proposed system mathematical model. Section 3 deliberates on the development of the fuzzy logic controller. Simulation results and discussions were presented in section 4 . Section 5 of the paper concludes the study. Acknowledgements and references were listed in sections 5 and 6.

\section{The Proposed Fresh Cassava Roots Storage System Model}

The proposed cassava storage system is depicted in Fig. 2. The mathematical model developed by [7] is adopted and applied to the proposed forced-air cassava roots crop storage system (with little modification) and use for the modeling of the forced-air storage system for temperature control in this study. They proposed a computer algorithm to approximate latent heat and sensible heat loads as well as the moisture loss and temperature distribution which occurs during the heating and cooling of heat and moisture releasing porous media. Heat and mass transfer in porous media has several important applications in domestic and industrial uses. Porous media system comprises of solid particles (called the matrix) and a stream of flowing air or water (as the fluid medium). Analysis of porous media have been employed in modeling heat and mass transfer which arises during storage and drying of various agricultural products as well as the aqueous transport of hazardous waste materials through soil surrounding landfill site[7]. Heat and mass transfer phenomena approximations within porous media are essential to designers of agricultural storage systems, dry and dehumidifying process facilities. A porous moisture absorbing material can be used in dehumidifying air in heating, ventilation and airconditioning (HVAC) systems[7]. Understanding the complex interaction of the various thermo-physical processes which takes place within and around the materials that form part of the porous medium is needed in estimation of heat and mass transfer in the medium.

In this study, the heat transfer model equation, which is based on one-dimensional airflow pattern through a bulk of load of cylindrical shaped food products is considered and the mass transfer model equation based on moisture loss by evaporation at the surface of the product driven by the difference between the vapour pressure on product surface and that of surrounding air is used.

The storage medium (see Fig. 2) comprises of commodity computational compartments (storage cells), enclosing fresh cassava roots in the rectangular-shaped structure and the conditioned air parcels passing through the four layers of commodity (fresh cassava roots) computational compartments. The schematic diagram of the proposed storage system is shown in Fig. 2. 


\subsection{The Heat Transfer Equation}

The governing one-dimensional transient heat transfer equation which describes the heat transfer in cylindrical coordinate system is as follows:

$$
(k / r) \partial(r \partial T / \partial r) / d r+\rho W=\rho c
$$

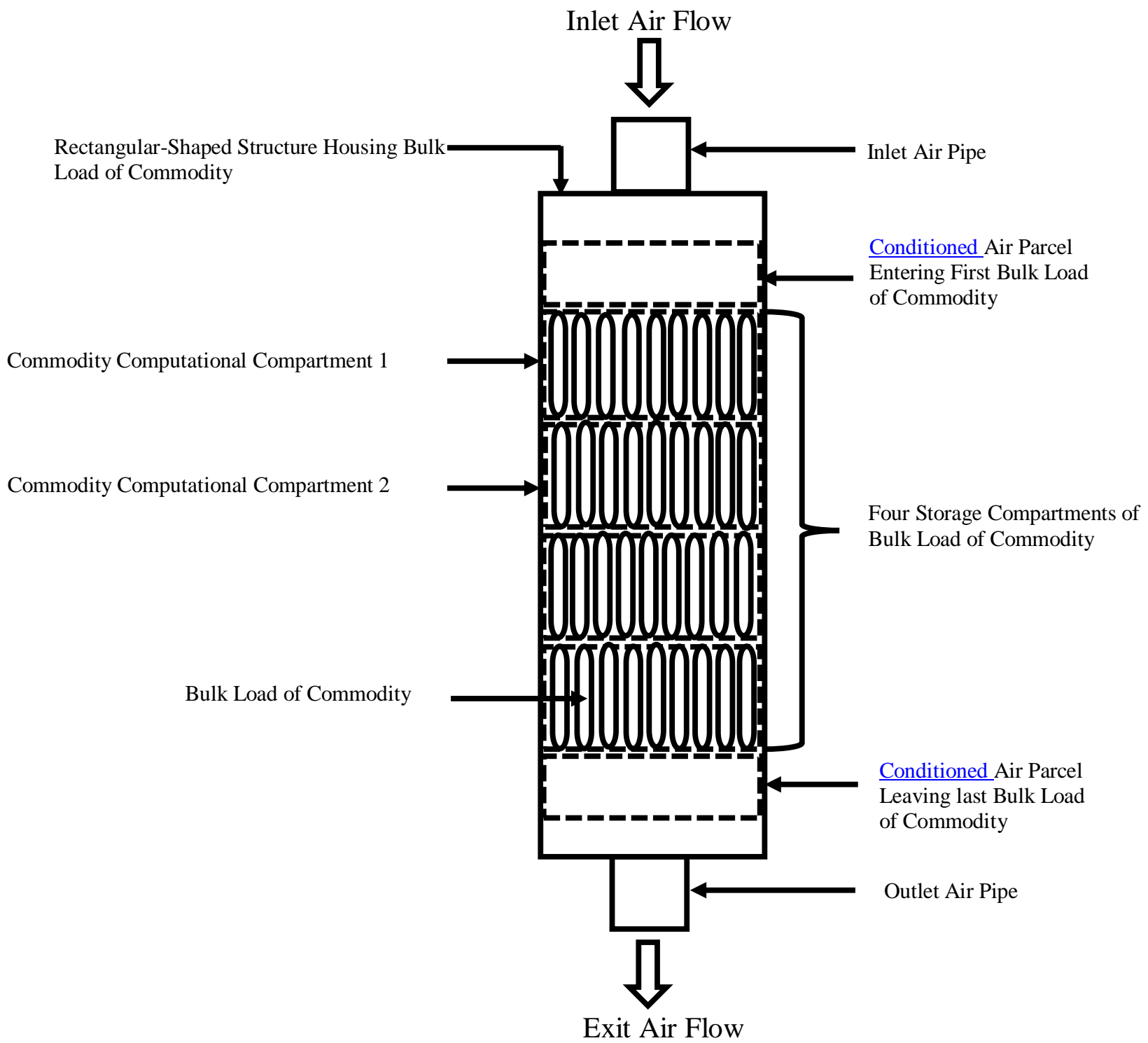

Fig. 2: Schematic Diagram of the Proposed Fresh Cassava Roots Crop Storage System.

Where: $k=$ Commodity thermal conductivity

$r=$ Radial direction

$T=$ Temperature of commodity

$\rho=$ Commodity density

$W=$ Heat of respiration generated per unit mass

$c=$ Commodity specific heat 


\subsection{The Mass Transfer Equation}

The transpiration process which occurs at the surface of the product involves the transport/removal of moisture from the cassava across its thin, porous skin. . Evaporation process takes place at the surface of the commodity followed by convective moisture transfer to the surrounding air. The driving force is the difference in water vapour pressure on the commodity surface and that of the surrounding air. The following equation shows the moisture loss from a single cassava root.

$$
m=k_{t}\left(P_{s}-P_{a}\right)
$$

$k_{t}$ Can also be modelled as:

$$
k_{t}=1 /\left[\left(1 / k_{s}\right)+\left(1 / k_{a}\right)\right]
$$

Where: $m=$ Transpiration rate per unit area of cassava root

$k_{t}=$ Transpiration coefficient

$P_{s}=$ Water vapour pressure at cassava root surface

$P_{a}=$ Water vapour pressure in surrounding air

$k_{s}=$ Skin mass transfer coefficient

$k_{a}=$ Air film mass transfer coefficient

The following are the assumptions made in the modeling process:

- Temperature variation within a unit cassava root restricted to radial direction

- The porous medium comprises of cylinders with uniform internal heat generation

- The cassava roots are of uniform dimension and distributed uniformly within the storage compartment

- The medium is of uniform porosity

- The water vapour pressure at the cassava roots surface is equal to the water vapour saturation pressure evaluated at the cassava roots' surface temperature

- The walls of the storage compartments are insulated to minimize temperature changes within the medium along the axis of flow

\subsection{The Operational Mechanism of the Storage System}

The schematic diagram of the proposed fresh cassava roots storage system which comprises of four storage compartments is shown in Fig. 2. Conditioned air from the cooling unit, modelled as air parcels, is passed into the storage system through commodity computational compartments via the inlet air pipe at the top. The air goes out of the system through the outlet air pipe. The interaction between the conditioned air and commodity computational compartments are extreme conditions which results in large temperature and moisture gradients within the storage system initially. Both gradients will continue to decrease with time until they become constant. Computations starts with the assumption that the computational time, $\mathrm{t}=0$ initially, temperature and moisture content of the commodity computational compartments and conditioned air are in equilibrium state. The cooling process begins with the first inlet plenum conditioned air (air parcel) moving downwards into commodity computational compartment 1 . Subsequently, all other air parcels will move to the next commodity computational compartment by displacing the initial one in similar manner. Conditioned air in the last compartment goes out from the bulk load into the outlet plenum of the cooling unit. Latent and sensible heat loads estimations is done using the properties of the air parcel in the last commodity computational compartment. Computation of mass transfer within each commodity computational compartment is done considering the moisture release rate $(\dot{m})$ for the time step, $d t$. In each air parcel, the mass fraction of water vapour (mf) is updated to reflect the effects of moisture release. Subsequently, heat transfer computation for the current time step within each compartment, begins with the calculation of the generated heat of respiration (W), 
heat transferred by products $(\mathrm{Q})$ to the air and the evaporative cooling due to moisture release. Then, within each storage compartments, both air and medium temperatures are updated to reflect the effects of heat generation, heat transfer and evaporative cooling. Hence, computations for this time-step are completed. In the next cycle, at time $\mathrm{t}=\mathrm{n} d t$, the computations are repeated starting from the beginning. Over time, each air parcel traverses the entire bulk load. The mass fraction of water vapour contained in each air parcel as it exits the bulk load is used to compute its latent heat load while the temperature of the exiting air serves to compute its sensible heat load.

\section{Development of Fuzzy Logic Controller for the Storage System}

Due to formation of large temperature and moisture gradient within the post-harvest storage system as a result of the interactions between conditioned air (air parcel) and the fresh cassava roots crop under storage, the storage process becomes highly nonlinear and complex in nature which demands a non-linear control approach. In such situations, fuzzy logic control, an intelligent and non-linear control technique which is based on computational intelligence is a most preferred choice.

Fuzzy logic control is a more successful feedback control technique which is simple to design and implement. It consists of a fuzzifier, rule base, an inference engine, and a defuzzifier all situated between preand post-processing blocks as shown in Fig. 4 [8]. FLC has been in use for several industrial applications since early 1970's[9]. FLC was applied successfully in various areas such as temperature control, process control, motion control, robotics, telecommunications, networking and in many more control systems [9-11].

TABLE I: The Proposed Fuzzy Logic Control Rules

\begin{tabular}{|c|c|c|c|c|c|}
\hline \multirow{2}{*}{$\mathrm{E}$} & \multicolumn{5}{|c|}{$\Delta \mathrm{E}$} \\
\cline { 2 - 6 } & $\mathrm{NB}$ & $\mathrm{NS}$ & $\mathrm{Z}$ & $\mathrm{PS}$ & $\mathrm{PB}$ \\
\hline NB & NB & NB & NB & NS & PZ \\
\hline NS & NB & NS & NS & Z & PS \\
\hline Z & NB & NS & Z & PS & PB \\
\hline PS & NS & Z & PS & PS & PB \\
\hline PB & Z & PS & PB & PB & PB \\
\hline
\end{tabular}

KEY: NB=Negative Big, NS=Negative Small, Z=Zero, PS=Positive Small, PB=Positive Big

\subsection{The Fuzzy Logic Controller Inputs and Output Variables}

The proposed temperature controller for the post-harvest storage system has two input variables, error "E" and change in error " $\Delta \mathrm{E}$ ", and one output variable " $\Delta U$ " which is the control input to the cooling unit. Triangular membership functions were employed in this study which produce minimal error in the desired output. Membership functions and steps rule base constitutes the computational structure of the FLC scheme. The proposed control rules were developed in the form of "IF-THEN" rules which governs the relationship between input and output variables The inputs to the controller, error $\mathrm{E}(\mathrm{k})$ and change in error $\Delta \mathrm{E}(\mathrm{k})$ are computed from the set-point value, $\mathrm{r}(\mathrm{k})$. The $\mathrm{k}$ denotes the discrete time. $\Delta \mathrm{U}(\mathrm{k})$ is fuzzy logic controller control output based on input variables. The 25 proposed fuzzy control rules are presented in Table I. The linguistic variables, NB, NS, Z, PS AND PB describes the membership functions for the inputs and output variables. Normalised values of inputs and output variables forms the universe of discourse ranging from -1 to +1 .

Temperature and relative humidity play a vital role in good post-harvest storage of fresh produce. However, in this study only storage temperature is considered. Hence, a fuzzy logic controller is proposed for temperature control for fresh cassava roots post-harvest storage system. The main task of the developed intelligent controller is to maintain optimum storage temperature $\left(3^{\circ} \mathrm{C}\right)$ of the storage system within acceptable range. The schematic of the controlled storage process involving the FLC, sensors, cooling unit and the (storage system) plant is illustrated in Fig. 3. 


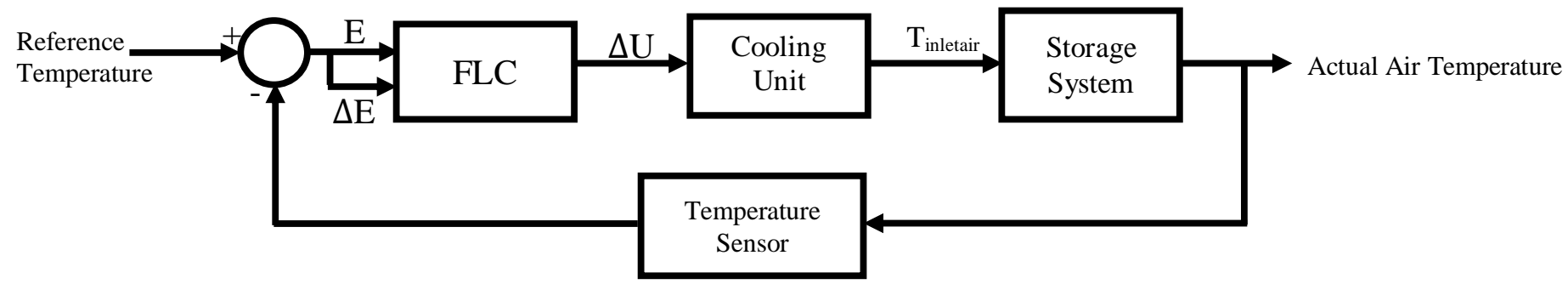

Fig. 3: Schematic Diagram of the Controlled Storage Process.

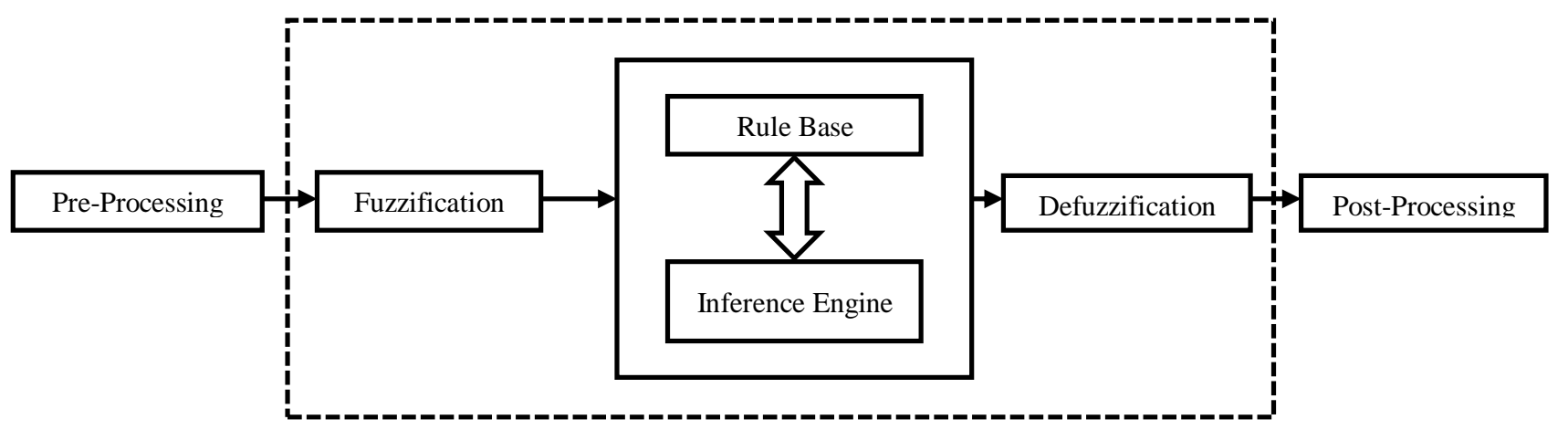

Fig. 4: Structure of Fuzzy Logic Controller.

\section{Simulation Results and Discussions}

For the purpose of this study, set-point tracking of the desired temperature and robustness towards input disturbance rejection by the designed FLC were carried out to evaluate the performance of the developed controller. Figures 5 and 6 shows the simulation results.

\subsection{Set-Point Tracking}

Fig. 5 illustrates the capability of the controller in tracking the desired reference (set-point) storage temperature. The controller was able to achieve the desired temperature within a settling time of about $3 \mathrm{~s}$, which shows its suitability for real-time implementation.

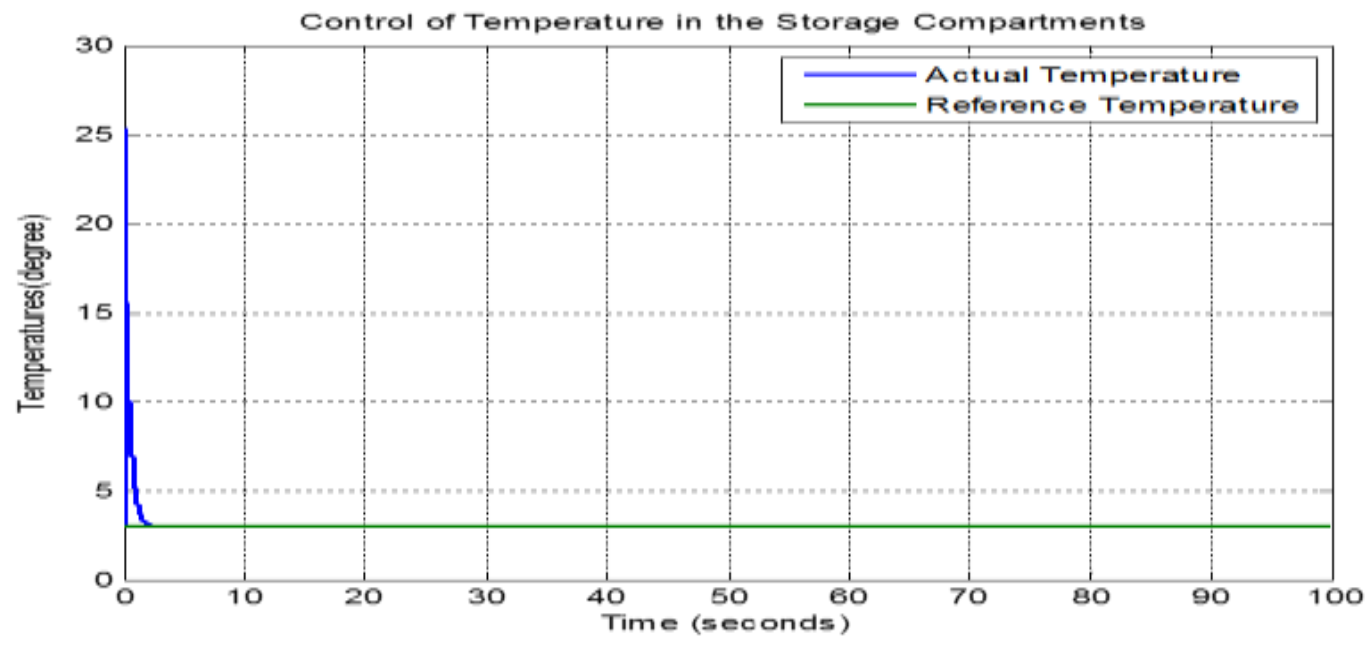

Fig. 5: System Response with FLC Indicating Set-Point $\left(3^{\circ} \mathrm{C}\right)$ Tracking. 


\subsection{Robustness Towards Disturbance Rejection}

A unit step input disturbance could occur in process of storage, for instance, sudden door opening of the storage system could cause ambient air to move into the system there by causing a change in the normal operation conditions of the storage system. Fig. 6 demonstrates the ability of the developed controller towards input disturbance rejection. As can be seen, at about 30 seconds of storage duration, disturbance test was carried out and the designed controller responds by adjusting the output appropriately.

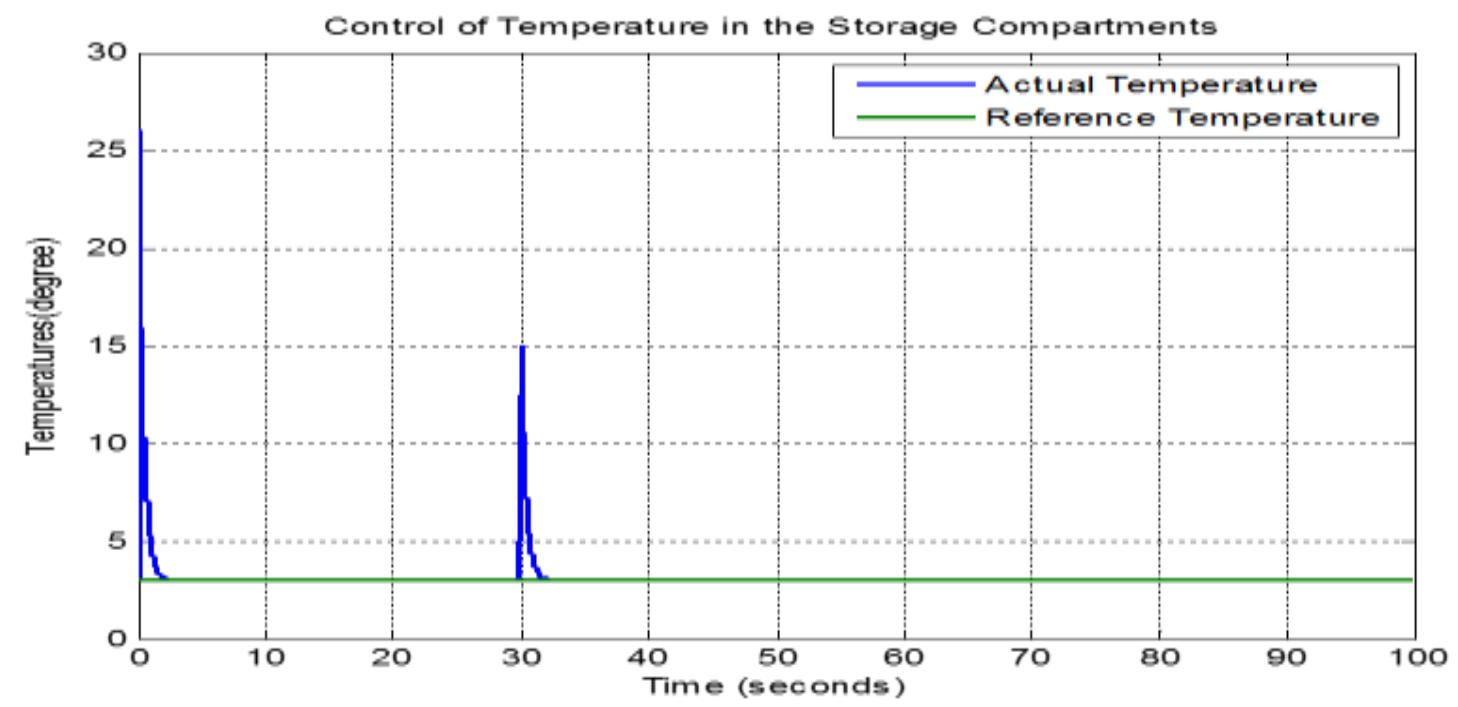

Fig. 6: System Response with FLC Indicating Step Input Disturbance.

\section{Conclusion}

A fuzzy logic controller (FLC) is proposed in this study due to its capability in tracking desired set-point temperature and ability to eliminate sudden unit step input disturbance in the fresh cassava roots storage system. The adopted mathematical model for spherical shaped food product was adapted for the cylindrical shaped cassava roots. Simulations studies was carried out using Matlab software environment and the results obtained clearly show that the FLC can track the desired set-point temperature within shortest possible settling time and at the same time rejects sudden input disturbances. This paper indicates the effectiveness of intelligent fuzzy logic controller in handling complex and highly non-linear systems, particularly for temperature control in fresh cassava roots post-harvest storage system.

\section{Acknowledgements}

The authors would like to acknowledge the assistance and financial support provided by Centre for Postgraduate Studies (CPS) IIUM towards making this work a success.

\section{References}

[1] A. Ambaw, M. Delele, T. Defraeye, Q. T. Ho, L. Opara, B. Nicolai, and P. Verboven, "The use of CFD to characterize and design post-harvest storage facilities: Past, present and future," Computers and Electronics in Agriculture, vol. 93, pp. 184-194, 2013.

http://dx.doi.org/10.1016/j.compag.2012.05.009

[2] M. Abdulazeez, M. Salami, and I. B. Tijani, "Intelligent Control for Automation of Yam Storage System Using Fuzzy Logic Controller," in Computational Intelligence, Modelling and Simulation (CIMSiM), 2011 Third International Conference on Computational Intelligence, Modeling \& Simulation, 2011, pp. 22-27.

http://dx.doi.org/10.1109/cimsim.2011.14 
[3] A. A. Oluwo, R. M. Khan, M. J. Salami, and M. A. Badran, "OPTIMIZED NEURAL NETWORK MODEL FOR A POTATO STORAGE SYSTEM," Journal of Agricultural \& Biological Science, vol. 8, 2013.

[4] O. A. Adeyinka, S. E. Momoh-Jimoh, K. M. Raisuddin, and A. A. Musa, "A review: Intelligent controllers for tropical food storage system," in Mechatronics (ICOM), 2011 4th International Conference On, 2011, pp. 1-7. http://dx.doi.org/10.1109/icom.2011.5937150

[5] A. D. Uchechukwu-Agua, O. J. Caleb, and U. L. Opara, "Postharvest Handling and Storage of Fresh Cassava Root and Products: a Review," Food and Bioprocess Technology, vol. 8, pp. 729-748, 2015.

http://dx.doi.org/10.1007/s11947-015-1478-z

[6] K. Simonyan, "Cassava post-harvest processing and storage in Nigeria: A review," African Journal of Agricultural Research, vol. 9, pp. 3853-3863, 2014.

[7] B. R. Becker, A. Misra, and B. A. Fricke, "Bulk refrigeration of fruits and vegetables Part I: theoretical considerations of heat and mass transfer," HVAC\&R Research, vol. 2, pp. 122-134, 1996.

[8] S. Ravi, M. Sudha, and P. Balakrishnan, "Design of intelligent self-tuning GA ANFIS temperature controller for plastic extrusion system," Modelling and Simulation in Engineering, vol. 2011, p. 12, 2011.

http://dx.doi.org/10.1155/2011/101437

[9] H. Mansor, M. Noor, S. Bahari, R. Ahmad, R. Kamil, F. S. Taip, and O. F. Lutfy, "Intelligent control of grain drying process using fuzzy logic controller," Journal of Food, Agriculture \& Environment, vol. 8, pp. 145-149, 2010.

[10] S. Khan, S. F. Abdulazeez, L. W. Adetunji, A. Z. Alam, and M. J. E. Salami, "Design and implementation of an optimal fuzzy logic controller using genetic algorithm," Journal of Computer Science, vol. 4, p. 799, 2008. http://dx.doi.org/10.3844/jcssp.2008.799.806

[11] A. Isizoh, S. Okide, A. Anazia, and C. Ogu, "Temperature control system using fuzzy logic technique," International Journal of Advanced Research in Artificial Intelligence (IJARAI), vol. 1, 2012. 